\title{
Molecular basis of hereditary fructose intolerance in Italy: identification of two novel mutations in the aldolase $B$ gene
}

\author{
R Santamaria, S Tamasi, G Del Piano, G Sebastio, G Andria, C Borrone, G Faldella, \\ P Izzo, F Salvatore
}

\begin{abstract}
We screened the aldolase $B$ gene in 14 unrelated Italian patients with hereditary fructose intolerance (HFI), and found two novel disease related mutations: a single nucleotide deletion in exon 2 ( $\triangle$ A20) that leads to an early stop codon, and a $\mathbf{C} \rightarrow T$ transition in exon 8 that substitutes an Arg with a Trp residue at codon 303 (R303W). $(\Im$ Med Genet 1996;33:786-788)
\end{abstract}

Key words: hereditary fructose intolerance; aldolase B; gene mutations.

Hereditary fructose intolerance (HFI) is an inborn error of carbohydrate metabolism caused by a deficiency of the glycolytic enzyme aldolase B. The frequency of HFI, inherited as an autosomal recessive trait, has been estimated at 1 in $20000 .^{1}$ Affected subjects, who are asymptomatic as long as they do not ingest foods containing fructose or sucrose, suffer from gastrointestinal pain, vomiting, and severe hypoglycaemia after fructose ingestion; liver damage and growth retardation can occur in the most severe forms. ${ }^{1}$

The most common disease related mutations in Europe are two missense mutations in exon 5, namely $\mathrm{A} 149 \mathrm{P}$ and $\mathrm{A} 174 \mathrm{D},{ }^{2}$ and $\mathrm{N} 334 \mathrm{~K}^{3}$ in exon 9. The other mutations are spread throughout the entire aldolase $B$ gene and some are rare or confined to single pedigrees. ${ }^{4}$

We have previously found that A149P and $\mathrm{A} 174 \mathrm{D}$ are also the most frequent mutations in Italian patients. ${ }^{5}{ }^{6}$ Moreover, two of our patients carried the MD $\Delta 4$ mutation, a rare $4 \mathrm{bp}$ deletion present in exon $4 .^{7}$ Here we describe two novel mutations, $\Delta \mathrm{A} 20$ in exon 2 and $\mathrm{R} 303 \mathrm{~W}$ in exon 8 , identified by a series of rapid polymerase chain reaction (PCR) based techniques: single strand conformation polymorphism (SSCP) analysis, direct sequencing, allele specific oligonucleotide hybridisation (ASO), and the amplification refractory mutation system (ARMS).

Total genomic DNA was extracted from peripheral blood leucocytes according to standard procedures ${ }^{8} ; 0.5-1 \mu \mathrm{g}$ of genomic DNA were amplified with 2.5 units $T a q$ polymerase, $200 \mu \mathrm{mol} / \mathrm{l}$ each of $\mathrm{dNTP}$, and 50 pmol of each primer, in a final volume of $100 \mu \mathrm{l}$, as follows: $95^{\circ} \mathrm{C}$ for two minutes, 30 steps at $94^{\circ} \mathrm{C}$ for 45 seconds, $55^{\circ} \mathrm{C}$ for $20 \mathrm{sec}-$ onds, $72^{\circ} \mathrm{C}$ for one minute, and a final elonga- tion at $72^{\circ} \mathrm{C}$ for five minutes. Amplified DNA products were examined by single strand conformation polymorphism analysis, carried out at $4^{\circ} \mathrm{C}$ on a $10 \%$ non-denaturing polyacrylamide gel with $5 \%$ glycerol, and visualised with the silver staining method. ${ }^{9}$ The ASO hybridisation conditions, the allele specific oligonucleotide sequences, and washing temperatures for the detection of A149P, A174D, and $\mathrm{N} 334 \mathrm{~K}$ mutations were as previously described. $^{236}$ ARMS analysis for the R303W mutation was carried out in the presence of an invariant antisense exon 8 primer, and a sense normal primer (5'-AACTAAGTTTCTCTTATCGAC-3') or a mutated primer (5'AACTAAGTTTCTCTTATCGAT-3'). The PCR products were electrophoresed on a $2 \%$ agarose gel and visualised after ethidium bromide staining.

The diagnosis of HFI in 14 patients was suspected because of characteristic symptoms after ingesting sweet tasting foods. Clinical diagnosis was confirmed by intravenous intake of fructose in eight patients, and by the reduced aldolase $B$ activity in liver biopsy specimens of three of those eight patients. These two tests were not performed in six patients, one of whom was clinically diagnosed as an infant and had a molecular diagnosis (A149P/Y203X) at the age of 30 years. The

Table 1 HFI mutations in 19 Italian patients

\begin{tabular}{|c|c|c|c|}
\hline Mutation & $\begin{array}{l}\text { Allele } \\
\text { frequency (\%) }\end{array}$ & Genotypes & $\begin{array}{l}\text { Genotype } \\
\text { frequency (\%) }\end{array}$ \\
\hline A174D & 29 & $\begin{array}{l}\text { A149P/ } \\
\text { A174D* } †\end{array}$ & 21 \\
\hline A149P & 29 & $\begin{array}{l}\text { A149P/ } \\
\text { A149P^ }\end{array}$ & 10.5 \\
\hline $\mathrm{MD} \Delta 4$ & 18.5 & $\begin{array}{l}\mathrm{A} 174 \mathrm{D} / \\
\Delta \mathrm{A} 20^{\star}\end{array}$ & 10.5 \\
\hline N334K & 5.2 & $\begin{array}{l}\mathrm{A} 174 \mathrm{D} / \\
\mathrm{MD} \Delta 4 \dagger\end{array}$ & 10.5 \\
\hline Y203X & 5.2 & $\begin{array}{l}\mathrm{A} 149 \mathrm{P} / \\
\mathrm{MD} \Delta 4^{\star}\end{array}$ & 10.5 \\
\hline R303W & 5.2 & $\begin{array}{l}\text { A174D/ } \\
\text { A174D }\end{array}$ & 5.3 \\
\hline$\Delta \mathrm{A} 20$ & 5.2 & $\begin{array}{l}M D \Delta 4 / \\
M D \Delta 4^{\star}\end{array}$ & 5.3 \\
\hline L256P & 2.7 & 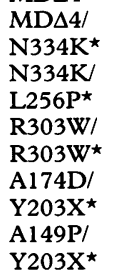 & $\begin{array}{l}5.3 \\
5.3 \\
5.3 \\
5.3 \\
5.3\end{array}$ \\
\hline
\end{tabular}

^ Data from this study.

t Data from ref 5 . 
genomic DNA of HFI patients was screened for the known HFI mutations and at least one of these mutations was found in all but one subject (table 1).

The molecular defect in the unidentified alleles in three subjects was detected by SSCP analysis and direct sequencing of the amplified exons. The SSCP analysis (data not shown) of
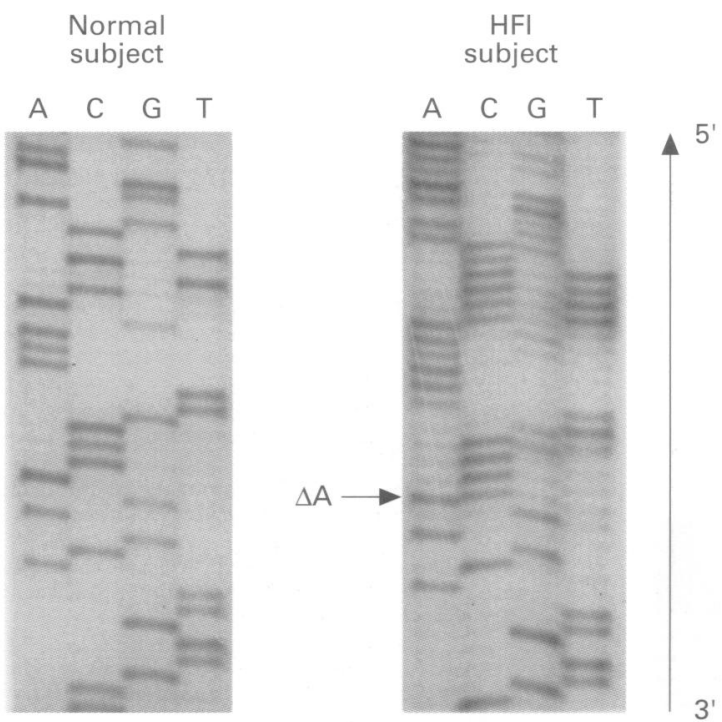

Normal sequence

\begin{tabular}{|c|c|c|c|c|c|}
\hline GAA & ATT & GCC & CAG & AGC & ATT \\
\hline Glu & $\mathrm{Ile}$ & Ala & GIn & Ser & Ile \\
\hline
\end{tabular}

$\triangle \mathrm{A} 20$ mutated sequence

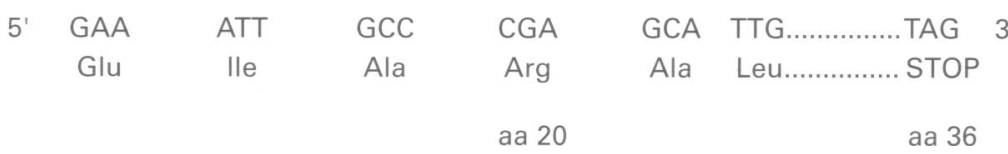

Figure 1 Sequence of double stranded exon 2 amplified DNA from a normal subject and an HFI patient. The deleted $A$ residue $(\Delta A)$ is marked by an arrow; normal and mutated sequences and codon translations are indicated. In the HFI subject bands $5^{\prime}$ to the $\triangle A$ position were altered by misalignment of the sequence from the two alleles; therefore the deletion of $A$ in one of them is deduced.
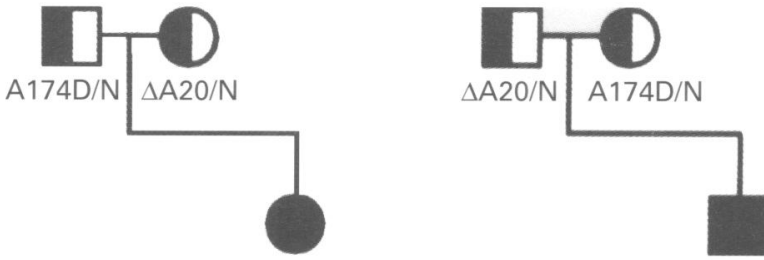

$\mathrm{A} 174 \mathrm{D} / \triangle \mathrm{A} 20$

$\triangle \mathrm{A} 20 / \mathrm{A} 174 \mathrm{D}$
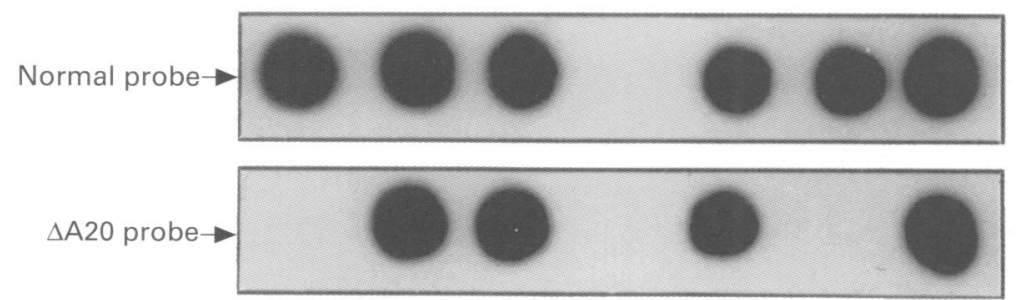

Figure 2 Segregation of the $\triangle A 20$ mutant allele in two families by ASO hybridisation of exon 2 amplified DNA. The normal (5'-GAAATTGCCCAGAGCATTGT'-3') or the mutated $\triangle A 20$ (5'-GAAATTGCCCGAGCATTGTT-3') oligonucleotide probes were used at the discriminatory washing temperature of $56^{\circ} \mathrm{C}$. The pedigrees of the two families and their genotype are reported in the upper section. exon 2 showed a shift in the electrophoretic mobility in two patients, already diagnosed by ASO as being heterozygous for the A174D mutation. Sequence analysis of amplified exon 2 showed in both these patients the deletion of the adenosine residue at the glutamine codon 20 (fig 1) that leads to a frameshift causing a premature stop at codon 36 . Both patients, compound heterozygotes for $\mathrm{A} 174 \mathrm{D} / \Delta \mathrm{A} 20$, showed liver failure characterised by hepatomegaly with diffuse steatosis of liver cells and moderate growth retardation. The segregation of this new mutation, designated " $\triangle \mathrm{A} 20$ ", in the families of the two unrelated probands was traced with the ASO method (fig 2).

In another patient, born to consanguineous parents, double stranded DNA sequence showed only in exon 8 a $\mathrm{C} \rightarrow \mathrm{T}$ transition in the first base of codon 303 (fig 3). This new missense mutation, designated "R303W", replaces the arginine 303 residue with a tryptophan residue. Arginine 303 is a critical residue, conserved in all vertebrate aldolases, essential for the interaction with the substrate and for the catalytic activity of the enzyme. ${ }^{10}$

Interestingly, the R303W mutation occurs at a CpG dinucleotide, a mutation "hot spot" region in the human genome. Clinical manifestations of the patient bearing this mutation were those classically observed in HFI patients. The aldolase $B$ activity in liver biopsy was undetectable, thus confirming the functional effect of this mutation on the aldolase $B$ enzyme.

We used ARMS to follow the segregation of the R303W mutation in the proband's family (fig 4). In the patient an amplified product was obtained only with the mutated primer, suggesting that she was homozygous for the R303W mutation. We were able to exclude the mutation in a 4 month old sister who had never been exposed to fructose. Unfortunately, no DNA sample from the proband's mother was available to follow the inheritance of the maternal mutant allele in the affected offspring; however, she must be a carrier of the R303W mutation because she is consanguineous with her husband.

The newly identified mutations, R303W and $\triangle \mathrm{A} 20$, were absent in the normal population as shown by the analysis of 40 chromosomes from 20 independent normal subjects.

Table 1 shows the HFI mutations found in all Italian patients studied so far by us, together with genotype constitution and frequency. The A149P and A174D mutations account for $58 \%$ of the mutant alleles, whereas the $\mathrm{N} 334 \mathrm{~K}$ and the less frequent mutations, MD $\Delta 4, \mathrm{~L} 256 \mathrm{P},{ }^{11}$ Y203X, ${ }^{12}$ R303W, and $\Delta \mathrm{A} 20$, account for the remaining $42 \%$. Interestingly, MD $\Delta 4$ accounts for $18.5 \%$ of mutant alleles; therefore it should be considered a frequent cause of HFI in Italy. The genotype of these patients indicates, as has also been shown in other populations, that compound heterozygotes prevail over the homozygotes.

Identification of new mutations in the aldolase $B$ gene and their analysis at the functional level will provide information about the structure-function relationships of the 


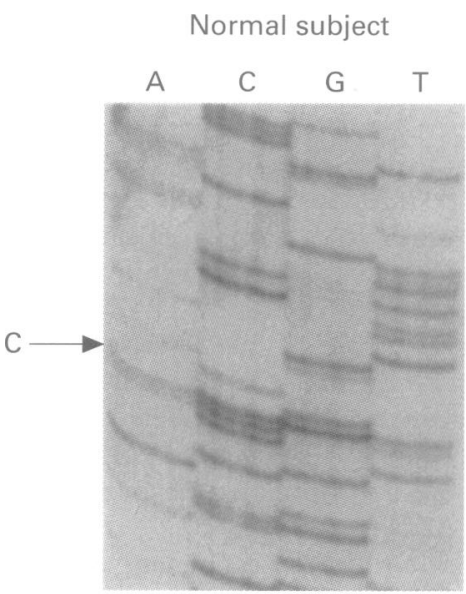

CGG (Arg303)
HFI subject

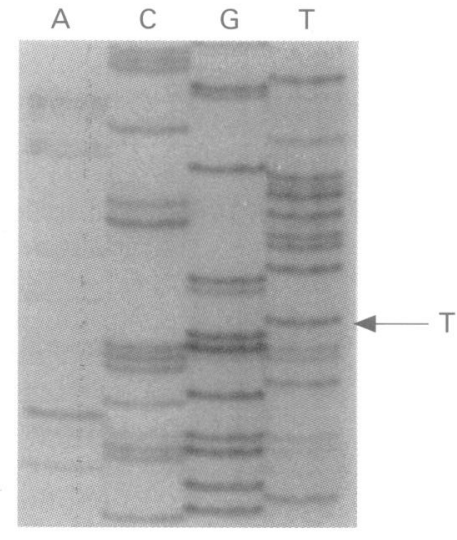

TGG (Trp303)
Figure 3 Sequence of double stranded exon 8 amplified DNA from a normal subject and from the HFI patient. Arrows indicate the position at codon 303 of the $C$ residue in the normal subject and the T residue in the HFI subject, respectively.

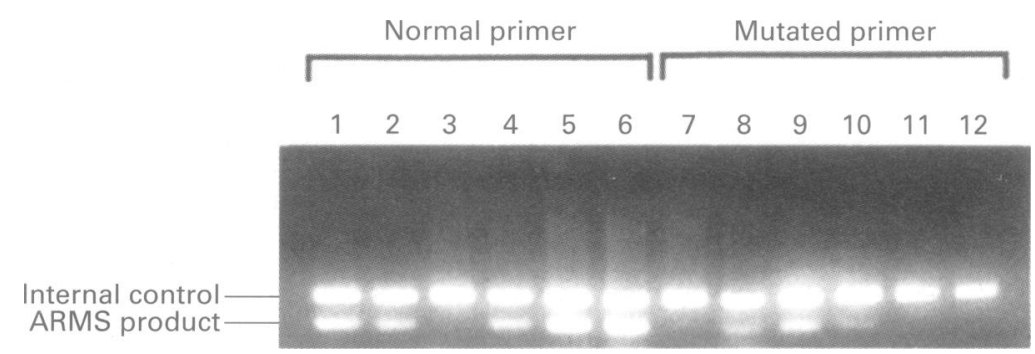

Figure 4 ARMS analysis for the R303W mutation in the presence of an invariant antisense exon 8 primer and a sense normal primer (lanes 1-6) or a sense mutated primer (lanes 7-12). In the patient (lanes 3 and 9) an amplified product was obtained only with the mutated primer; amplified products with normal and mutated primers were obtained in the father (lanes 2 and 8) and in a healthy sister (lanes 4 and 10); an amplified product only with normal primer was obtained in a 4 month old sister (lanes 5 and 11); DNA from healthy control subjects (lanes 1 and 7,6 and 12). The ARMS product is $195 \mathrm{bp}$ and the internal control 290 bp. The control without DNA was negative (data not shown). enzyme, and could have an impact on molecular epidemiological studies, early diagnosis of HFI, and screening of carriers in affected families, who would otherwise be difficult to detect.

This work was supported by grants from MURST, CNR (Target Project "Biotechnology and Bioinstrumentations" and "Ingegneria Genetica"), Rome, Regione Campania "Ricerca Sanitaria Finalizzata", and "Agen-Sud", Rome, Italy.

1 Gitzelmann R, Steinmann B, Van den Berghe G. Disorders of fructose metabolism. In: Scriver CR, Beaudet AL, Sly WS, Valle D, eds. The metabolic and molecular bases of inherited disease. 7th ed. New York: McGraw-Hill, 1994:905-34.

2 Cross NCP, de Franchis R, Sebastio G, et al. Molecula analysis of aldolase $\mathrm{B}$ genes in hereditary fructose intolerance. Lancet 1990;335:306-9.

3 Cross NCP, Stojanov LM, Cox TM. A new aldolase B variant, $\mathrm{N} 334 \mathrm{~K}$, is a common cause of hereditary fructose intolerance in Yugoslavia. Nucleic Acids Res 1990;18:1925.

4 Cox TM. Aldolase $\mathrm{B}$ and fructose intolerance. FASEB $\mathcal{F}$ 1994;8:62-71.

5 Sebastio G, de Franchis R, Strisciuglio P, et al. Aldolase B mutations in Italian families affected by hereditary fructose intolerance. 7 Med Genet 1991;28:241-3.

6 Santamaria R, Scarano MI, Esposito G, Chiandetti L, Izzo $\mathrm{P}$, Salvatore F. The molecular basis of hereditary fructose intolerance in Italian children. Eur f Clin Chem Clin Biochem 1993;31:675-8.

7 Dazzo C, Tolan DR. Molecular evidence for compound heterozygosity in hereditary fructose intolerance. $\mathrm{Am} \mathscr{J}$ Hum Genet 1990;46:1 194-9.

8 Miller SA, Dykes DD, Polesky HF. A simple salting out procedure for extracting DNA from human nucleated cells. Nucleic Acids Res 1988;16:1215.

9 Budowle B, Chakraborty R, Giusti AM, Eisenberg AJ, Allen RC. Analysis of the VNTR locus DIS 80 by the PCR followed by high-resolution PAGE. Am of Hum Genet 1991;48:137-44.

10 Gamblin SJ, Davies GJ, Grimes JM, Jackson RM, Littlechild JA, Watson HC. Activity and specificity of human aldolases. F Mol Biol 1991;219:573-6.

11 Ali M, Sebastio G, Cox TM. Identification of a novel mutation (Leu 256 $\rightarrow$ Pro) in the human aldolase B gene associated with hereditary fructose intolerance. Hum Mol Genet 1994;3:203-4.

12 Ali M, Rosien U, Cox TM. DNA diagnosis of fatal fructose intolerance from archival tissue. $Q \Im \mathrm{Med} 1993 ; 86: 25-30$. 